\title{
Nutritionally adequate and culturally relevant to the eating patterns adapted to meet the dietary guideline 2005 recommendations for University of Arkansas students at Pine Bluff
}

\author{
Ying Hua Gao-Balch*, Felicia D Taylor Waller and Brenda Simmons \\ Department of Human Science, University of Arkansas at Pine Bluff, 1200 North University Drive, Pine Bluff, Arkansas 71601, USA
}

\begin{abstract}
Nutritionally adequate and culturally relevant to the eating patterns adapted to meet the Dietary guideline 2005 recommendations method on the improvement of body weight. A 12 -month study completed by focused on interventions to improve physical activity and health through walking teams led by supportive coaches, selfmonitoring, and monthly nutrition and physical education sessions involved regular aerobic, strength training, and stretching based on the Trans theoretical model. Of 80 participants 10\% were normal weight, 22\% were overweight, and 57\% were obese. Ninety-nine percent were African American and 97\% were women. The average age was 21 years. Results indicated that documented from the beginning to the end of the intervention season indicated that (58\%) of the original students who returned for follow-up assessments had a weight loss was $5 \%$ in $52 \%$ of the students, weight gain in $6 \%$ in $18 \%$ of the students. Significant weight loss was $10 \%$ in $11 \%$ of the students, it was not significant weight loss was $2 \%$ in the $19 \%$ of the students with during intervention 12 months season ( $p<0.0001$ ). Multicomponent university students obesity intervention effectively program may need further more research.
\end{abstract}

\section{Introduction}

Currently more than $65 \%$ of American adults are overweight or obese, making improvements in this area arguably one of the most important health challenges of our time. Most obesity research to date has focused on biomedical aspects rather than on the importance of diet and physical activity behaviors in maintaining a healthy body weight. There is a need to identify new strategies that will enhance adherence to the healthful food and physical activity patterns set forth in the Dietary Guidelines for Americans, 2005 (DG). There is also a need to adapt established DG eating patterns, for the Lower Mississippi Delta population and to test their effectiveness in preventing obesity and related diseases. Minimal research has addressed sustained prevention and intervention strategies to prevent obesity and related diseases for African Americans, especially college/university students [1-6].

The increase in obesity is moving steadily away from the Healthy People 2010 goals of $15 \%$ prevalence of obesity in adults and 5\% prevalence in children. Overweight continues to be a serious health concern for children, adolescents and young adults and is a leading predictor of obesity in adulthood increasing the prevalence of chronic illness [7-10]. The American Dietary guideline was designed to improve the dietary and physical activity patterns of all Americans. However, research conducted found that participants' knowledge of the 2000 DG was lacking in the areas of food composition, healthier eating, and the relationship between diet and health, and food labels [11]. Since 2000, evidence of the public's understanding, perception and exposure to the 2005 recommended the American dietary guideline remains elusive in the literature. Additionally, as many as 90 million Americans lacked basic skills needed to access, understand, and use health information and services to make healthy dietary choices [12]. However, research has documented knowledge of the DG by college/university students and Americans and has found nutrition literacy to be less than adequate $[11,12]$.

Education, health, and nutrition disparities are well documented and continue to have a significant impact in the LMD; however, understanding nutrition literacy in the LMD and the role it may play in the adoption of the DG eating patterns and their effectiveness in preventing obesity and related diseases is paramount for targeted populations. Nutrition literacy has been defined by as "the capacity to obtain, process, and understand basic nutrition information needed to make appropriate dietary decisions [13]." They further report that no known published research has examined the nutrition literacy status of residents residing in the LMD region. Nutrition education and physical activity interventions that focus on healthy eating based on the DG and the relationship between diet and health in the LMD with the aim of preventing many new cases of diet-related chronic disease make it worthwhile in the long term for college/university students [14].

Eating habits are hard to change because they are part of an individual's acculturation; therefore, new habits must be learned. Eating patterns of different ethnic groups have also confused our understanding of the relationship between nutrient intake and chronic

Correspondence to: Ying Hua Gao-Balch, Department of Human Science, University of Arkansas at Pine Bluff, 1200 North University Drive, Pine Bluff, Arkansas 71601, USA, E-mail: gaobalchy@uapb.edu

Key words: nutrition, body weight loss, dietary guideline

Received: May 11, 2015; Accepted: June 10, 2015; Published: June 13, 2015 
diseases, and obesity. Efforts to reduce overweight and obesity have not included the role of nutritional literacy, but rather educational activities that have focused on food preparation and encouraging families and patients with chronic illnesses or who are attempting to lose weight to modify sodium, fat, and sugar content of familiar foods. Other areas included cutting calories and eating smaller portions, and revising the Food Pyramid; yet obesity continues to negatively impact minority communities. Studies report that African Americans have the widest deviance from the current nutritional guidelines compared with non-Hispanics whites, and raise the question of why AfricanAmerican (non-Hispanic Blacks) eating patterns have not equilibrated with mainstream eating pattern after so many generations. In many instances, culturally familiar ways of eating are defined as "barriers" and viewed as "non-adherent [15]." Essential motivators of eating patterns, such as flavor, price, tradition, and emotional and social meanings, should not be ignored or devalued in dietary guidance but considered essential in minority groups [15].

The success of many interventions aimed at motivating healthy behavior change will be measured by the degree to which cultural and contextual factors are taken into consideration in the intervention design. Health behavior and health belief may be influenced by culture, which is a major determinant of how people understand, interpret, and respond to various experiences [16]. This is even more evident in obesity interventions because several individual weight management programs have failed to achieve long-term reduction in weight through diet and other interventions targeted at the individual [17]. "The contexts that have established and nurtured obesity are systemic and structural, hence the need to turn to culture" [18].

Culture influences most aspects of human endeavors including why, what, how, and with whom we eat $(28,32)$. People within a particular community often share common markets, restaurants, and foods. Therefore, over a course of time what, where, how, and when they eat becomes one of the main identifiable characteristics of a community and hence a dependable way of characterizing and possibly distinguishing a community's culture. Once ingrained into a culture, eating behaviors then become a way of expressing cultural identity. For instance, in many collectivist cultures, eating is an important element of social gatherings and it is considered impolite to refuse food especially when presented by a hostess. Similarly, when receiving a guest, it is considered impolite not to offer generous amounts of food [19]. The cultural differences in eating attitudes and behaviors imply that an individual's cultural orientation may influence the attitudes and behaviors around healthy eating. For example, research has shown that collectivists-individualists' orientation influences the effectiveness of various healthy eating interventions [20]. Therefore, it might be difficult to design an effective intervention for motivating healthy eating without taking the cultural orientation of the target group into consideration.

African Americans have the widest deviance from the current nutritional guidelines compared with non-Hispanics whites, and raises the question of why African-American (non-Hispanic Blacks) eating patterns have not equilibrated with mainstream eating pattern after so many generations [15]. In many instances, culturally familiar ways of eating are defined as "barriers" and viewed as "non-adherent." Essential motivators of eating patterns, such as flavor, price, tradition, and emotional and social meanings, should not be ignored or devalued in dietary guidance but considered essential in minority groups [15].

Researchers have found that despite the poor eating habits that manifest in college, the overall mean caloric intake in college freshmen may actually decrease [21-23]. Yet at the same time, body weight tends to increase during the college years, suggesting that physical activity must also be decreasing during the transition from high school to college [24]. Intervention programs directed towards college/university students focused primarily on strategies intended to decrease the body weight, through behavioral counseling, nutrition education without physical activity, or decreased or modified dietary intake with or without physical activity [18-25]. Although these studies addressed dietary interventions, the majority found that physical activity and lifestyle were important determinants in the outcomes. students attending the University of Kansas, Lawrence, were overweight and engaged in less than healthy dietary habits, such as low fruit and vegetable intake, low fiber intake, and low physical activity, suggesting the need for greater attention to diet and exercise interventions in this population. More importantly, the findings suggested that physical activity levels may decrease as students become older [18]. In comparison, study participants who started the National Heart, Lung, and Blood Institute's Growth and Health Study at ages 9-10 years had a significant decline in physical activity by ages $[18,19]$. Similarly, the greatest deterioration in physical activity has been observed between the ages of 15 and 18 years, and a continuous decline is common between 18 and 29 years of age [20]. Overall, the most significant finding was a decrease in physical activity. In some studies students did not have an increase in caloric intake but gained weight. Issues that motivated the student's decision to decrease physical activity need further attention.

A nutrition education program, without physical activity, that addressed issues, such as a personal diet plan based on an appropriate calorie level, label reading, behavior change techniques, and portion control using the Plate Model to design healthy meals that fit their meal plan [22]. The importance of exercise is only discussed in this program. A student not demonstrating success was offered behavioral counseling. Effectiveness of the program was evaluated through nutrition knowledge pre-test and post-test relevant to the nutrition content areas. The premise of this program is that an increase in nutrition knowledge and skill development will contribute to a healthier final 24-hour dietary recall when compared to the first recall. Effectiveness of these interventions were not discussed; however, the university offers specific interventions for obesity as a part of services students commonly receive, i.e., meals, transportation, lab fees, which may be an option for other colleges/universities.

The African-American women who are overweight may not perceive themselves to be overweight; increasing the importance of what role image has in weight maintenance [6]. Image is extremely important for this age group and may have a significant influence in their decisions to participant in physical activity. Socially acceptable forms of physical activity may be fewer for women than men, particularly in some ethnic groups. Social concerns may include how exercise affects one's hairstyle or image of femininity, as well as the possible displeasure of spouses or other household members, because exercise may be perceived as taking a woman away from family responsibilities [24].

Another risk factor associated with overweight and obese in college/ university students postulated by Desai et al. (2008) is disordered eating [17]. The author notes that although the eating behaviors leading to obesity are not technically considered disordered eating behaviors, it is important to note that overweight individuals do exhibit disordered eating symptoms, such as feelings of ineffectiveness, perfectionist attitudes, low introspective awareness, low self-esteem, emotional eating, and depressive symptoms. These psychological concerns have 
implications for the treatment of overweight in that a simple dietary intervention or physical education course may not be sufficient to generate a sustainable reduction in young adult overweight and obesity. The study conducted by Desai et al. [17] found that overweight and obesity are associated with distorted eating behaviors and perceptions. Specifically, a preoccupation with food, feeling that food controlled one's life, the desire to be thinner, and bingeing and dieting behaviors were all positively associated with overweight and obesity.

This study was evaluate the effectiveness of the weight loss initiative of approach, how the nutritionally adequate and culturally relevant to the eating patterns adapted to meet the Dietary guideline 2005 recommendations for University of Arkansas at Pine Bluff

\section{Methods}

Participants was recruited through university Arkansas at pine bluff to the development of the program, to collect baseline data that was analyzed and used to develop the "Eating to Live" program. Participants (80) of interview was developed that includes questions that elicit strategies, perceptions, and concerns related to the topics and activities to be used in developing the "Eating to Live" curriculum and ultimately the intervention. Questions were elicited perceptions and attitudes. The participants' interview was allowed the researchers to gather information from more than one person in less time. This approach also provides checks and balances that weed out false or extreme views. An incentive of $\$ 20$ per person was paid to each participant participating in each session. The participants were address dietary and physical activity patterns, knowledge of the food groups, food labels, American Dietary Guidelines for Americans, 2005.

Dietary records were proposed during the intervention only. Although they was more extensive, the participant was record the foods and beverage and the amounts of each consumed over 3-4 days. The meal was recorded at the time of the eating occasion. This was done online monkey service. The participants were asked to take a picture of the meals eaten (breakfast, lunch and dinner). The dietary record method has the potential for providing quantitatively accurate information on food consumed during the recording period. Information needed for the Plate Model module was collected by photographs (via cell phone or digital cameras) of foods eaten by the participants to document current eating patterns. The photographs was used to validate the information collected in the recalls and records; i.e., food combinations, and portion sizes, amount of fat, carbohydrate, vegetables, and proteins consumed $[22,25]$. Participants was using cell phones was send data to investigators by email, or an internet computer download site. These photographs were potentially representing culturally relevant meals for this population and were used in developing the educational content to be used in the intervention. Meals from menus based on the American dietary guideline, prepared by the Department of Human Sciences and UAPB cafeteria was tasted by the participants to test acceptability in order to better understand how the American dietary guideline was adapted for this intervention.

The "Eating to Live" program was a culturally adapted 12-month weight loss intervention designed based on adoption of the American dietary guidelines 2005 , This programs was enhanced with adapted DG recommendations and strategies, based on the baseline data collected from group interviews, 24-hr dietary recalls, the current plates of food consumed by the students, photographs of the current meals and surveys of the 'food availability' environment. The current plates of food consumed was analyzed for combination of foods and portion sizes, Photographs of food combinations and portion sizes was used to modify the food plates based on recommendations in the American dietary guideline. Modified food plates were compared to the American dietary guideline recommend.

\section{Data analysis}

Participants in the initial phase were asked to complete four 24hour dietary recalls over a period of 2 weeks (two per week). Foods and beverages in the preceding 24 hours or in the preceding day was recorded by the investigator (dietitian) to clarify discrepancies or probe for forgotten foods or meals. The 24-hour recalls was use standard protocols for the Nutrition Data System for Research (NDSR); DSAM systems (software, version 4.06). The NDSR was used to analyze the 24-hour recalls used in the development phrase because there was a lower respondent burden than dietary records, which was used in the intervention. The NDSR protocol uses a multiple pass approach to reduce underreporting and to assist further standardization of the interviews. Data was collected by a nutritionist certified as a NDSR trainer (Nutrition Coordinating Center 2008).

Qualitative data was analyzed using NVivo7 software to find patterns in the data, and enable the researchers to display and analyze relationships in the data. Individual's eating and physical activity patterns were compared to the American dietary guideline recommendations.

\section{Results}

A 12 -month study completed by focused on interventions to improve physical activity and health through walking teams led by supportive coaches, self-monitoring, and monthly nutrition and physical education sessions involved regular aerobic, strength training, and stretching based on the Trans theoretical model [21] Table 1. Of 80 participants $10 \%$ were normal weight, $22 \%$ were overweight, and $57 \%$ were obese. Ninety-nine percent were African American and $97 \%$ were women. The average age was 21 years. Results indicated that documented from the beginning to the end of the intervention season indicated that (58\%) of the original students who returned for follow-up assessments had a weight loss was $5 \%$ in $52 \%$ of the students, weight gain in $6 \%$ in $18 \%$ of the students. Significant weight loss was $10 \%$ in $11 \%$ of the students, it was not significant weight loss was $2 \%$ in the $19 \%$ of the students with during intervention 12 months season ( $p<.0001)$. Conclusions: A 12-month multicomponent school-based obesity intervention program may be effectively used to decrease body weight.

\section{Discussion}

During behavior intervention, we were using the conceptual framework guiding the development and delivery of the intervention was the Trans theoretical Model also known as the stages of change

Table 1. 12 Months Intervention Results

\begin{tabular}{|l|c|}
\hline Participants & UAPB Students \\
\hline Normal weight & $11 \%$ \\
\hline Overweight & $32 \%$ \\
\hline Obese & $57 \%$ \\
\hline After 12 months intervention & $5 \%$ in $52 \%$ \\
\hline Weight loss & $6 \%$ in $18 \%$ \\
\hline Weight gain & $10 \%$ in $11 \%$ \\
\hline Significant weight loss & $2 \%$ in $19 \%$ \\
\hline Not significant weight loss & $\mathrm{P}<.0001$ \\
\hline
\end{tabular}


Gao-Balch YH (2015) Nutritionally adequate and culturally relevant to the eating patterns adapted to meet the dietary guideline 2005 recommendations for University of Arkansas students at Pine Bluff

Table 2. DOPRP POST-Test 2013.

\begin{tabular}{|l|c|c|}
\hline \multicolumn{3}{|l|}{ Which of the following courses did you find most useful? } \\
\hline Answer Options & Response Percent & Response Count \\
\hline BMI/Dietary guidelines (Gray) & $47.9 \%$ & 23 \\
\hline Eating to Lose Weight (Ying) & $56.3 \%$ & 27 \\
\hline Healthy Eating (Gray) & $60.4 \%$ & 29 \\
\hline Stress Management (Taylor-Waller) & $52.1 \%$ & 25 \\
\hline Cooking Class-Breakfast burrito & $35.4 \%$ & 17 \\
\hline Cooking Class-Vegetable fried rice & $12.5 \%$ & 6 \\
\hline Cooking Class Makeup (Shrimp tacos) & $2.1 \%$ & 1 \\
\hline & answered question & \\
\hline & skipped question & \\
\hline
\end{tabular}

model [26]. This model emphasizes behavior change as an individual move through the stages of readiness of change: pre-contemplation (not thinking about changing in the next 6 months), contemplation (thinking about changing within the next 6 months), preparation (intending to take action in the near future, usually 1 month), action (specific changes have been made), and finally maintenance (working to prevent relapse). Determination of individual readiness for change was evaluated using a forced-choice questionnaire based on the stages of the change process. The application of the Trans theoretical Model [26] was similar to the Health Stages program for older adults used. As in Health Stages, the Eating to live program has several components: a) a comprehensive curriculum, b) an implementation strategy, and c) a program evaluation. TTM is the organizing concept for the program/intervention and the TTM concepts was integrated into program planning, curriculum development, and program evaluation. Program planning: According to TTM, people are in different stages of readiness to change any given health behavior. As a result, different types of information and interventions were needed for people who were in different stages. For example, people may be in maintenance for one behavior, like exercise, but in Contemplation for eating a low fat diet. The TTM encourages the researcher to develop a variety of ways to reach out to the participants. Staging of the participants for health behaviors provides a way to target needed interventions to the participants.

To assess the effectiveness of this obesity prevention intervention program using nutritionally adequate and culturally relevant to the eating patterns adapted to meet the Dietary guideline 2005 recommendations approach in decreasing overweight at a period of 12 months among university students. Our study finding confirmed necessity of school building-level implementation of the intervention, showing some of a significant improvement in students' health status and healthy behaviors. Given a significant role of schools due to an efficient accessibility to a variety of resources intervention implementation at level of individual to better tailor the intervention programs and take ownership over planning programs more fit to their students' needs, leading to more promising outcomes in health promotion by Figures 1 - 3. The findings of the present study strengthen that an intervention which is committed to making school environments conducive to students' healthy behaviors and changing students' individual behavior and norms may help students maintain or reduce their overweight over a relatively long time span by Figures 1-3.

Limitations of this study should be noted. First, a single measurement on a single occasion may be susceptible to measurement errors. Second, although biometric data such as height, and weight were

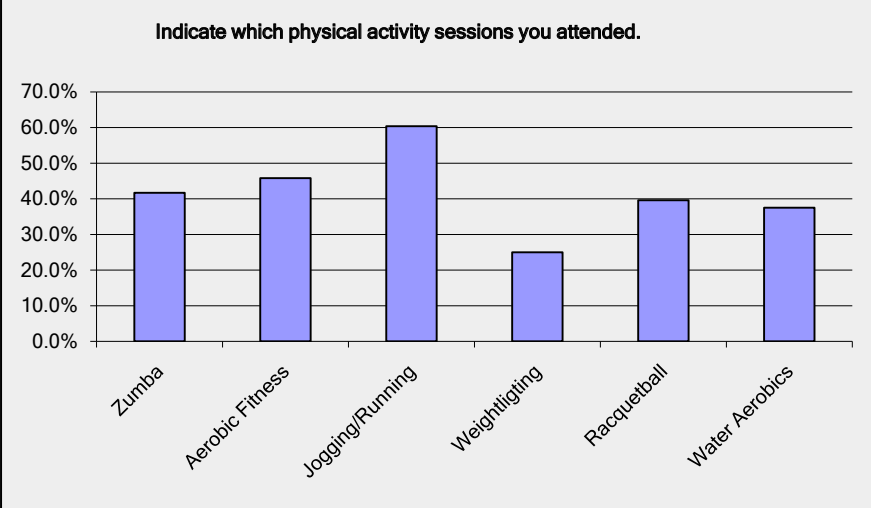

Figure 1. Investigation for physical activity sessions.

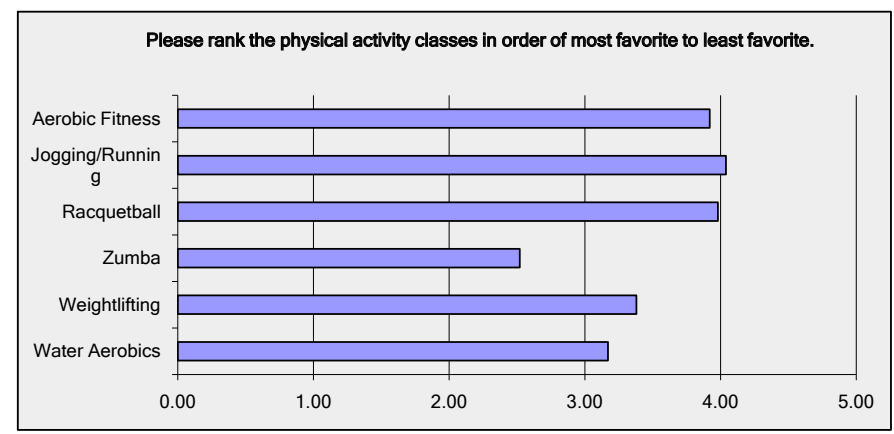

Figure 2. Investigation for physical activity classes.

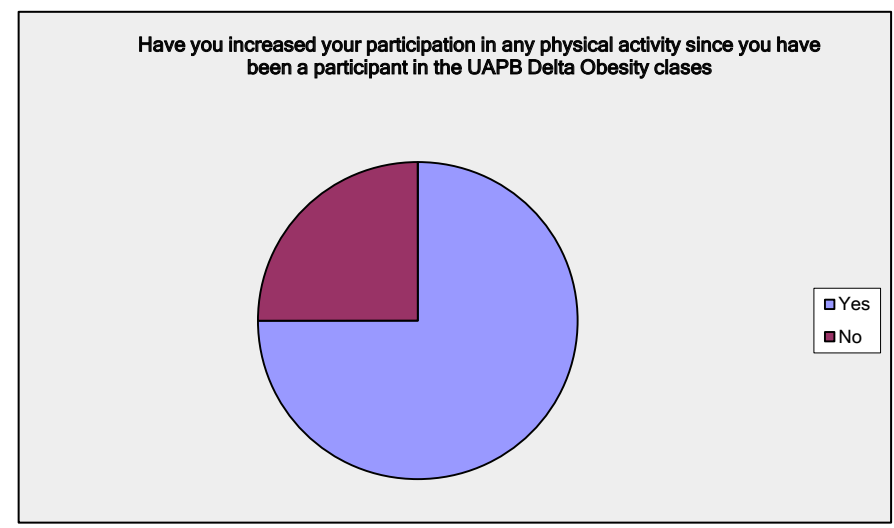

Figure 3. Investigation for physical activity in the UAPB Delta Obesity classes.

objectively measured at each data collection point for the entirety of participants, behavioral measures were collected using surveys. Thus, self-reports on behavioral measures might have confounded study findings. Another finding is participants of retention need further a discussion more by Table 2 . Despite these limitations, This study was evaluate the effectiveness of the weight loss initiative, university of Arkansas at Pine Bluff obesity prevention intervention based on the Dietary guideline for American of approach may be effectively used to decrease body weight. Further investigation would be warranted to evaluate effectiveness of multicomponent intervention program to a targeted population for efficacy of the desirable no pharmacological treatments for university students to maintain healthy weight. 
Gao-Balch YH (2015) Nutritionally adequate and culturally relevant to the eating patterns adapted to meet the dietary guideline 2005 recommendations for University of Arkansas students at Pine Bluff

\section{Sponsoring agency}

USDA ARS grant-Obesity in College/University Students 20092013.

\section{References}

1. Fitzgibbon ML, Stolley M, Schiffer L, Sharp L, Singh V, et al. (2008) Obesity Reduction Black Intervention Trial (ORBIT): Design and baseline characteristics. Journal of Women's Health 17: 1099-1110. [Crossref]

2. Svetkey LP, Erlinger TP, Vollmer WM, Feldstein A, Cooper LS, et al. (2005) Effect of lifestyle modifications on blood pressure by race, sex, hypertension status, and age. $J$ Hum Hypertens 19: 21-31. [Crossref]

3. Wing RR (2000) Maintenance of behavior change in cardiorespiratory risk reduction: Introduction to Proceedings from the National Heart, Lung, and Blood Institute conference; 1988 Jul 6-7; Bethesda (MD). Health Psychology 19: 3-4.

4. Kimm SY, Glynn NW, Kriska AM, Fitzgerald SL, Aaron DJ, et al. (2000) Longitudinal changes in physical activity in a biracial cohort during adolescence. Med Sci Sports Exerc 32: 1445-1454. [Crossref]

5. Garner D, Wooley S (1997) Confronting the failure of behavioral and dietary treatments for obesity. Clinical Psychology Review 11: 573-578.

6. Yancey AK, Simon PA, McCarthy WJ, Lightstone AS, Fielding JE (2006) Ethnic and sex variations in overweight self-perception: relationship to sedentariness. Obesity (Silver Spring) 14: 980-988. [Crossref]

7. Colditz GA, Willett WC, Rotnitzky A, Manson JE (1995) Weight gain as a risk factor for clinical diabetes mellitus in women. Ann Intern Med 122: 481-486. [Crossref]

8. McTigue KM, Hess R, Ziouras J (2006) Obesity in older adults: a systematic review of the evidence for diagnosis and treatment. Obesity (Silver Spring) 14: 1485-1497. [Crossref]

9. Truesdale KP, Stevens J, Lewis CE, Schreiner PJ, Loria CM, et al. (2006) Changes in risk factors for cardiovascular disease by baseline weight status in young adults who maintain or grain weight over 15 years: The CARDIA Study. International Journal of Obesity (London) 30: 1397-1407. [Crossref]

10. Lloyd-Jones DM, Liu K, Colangelo LA, Yan LL, Klein L, et al. (2007) Consistently stable or decreased body mass index in young adulthood and longitudinal changes in metabolic syndrome components: the Coronary Artery Risk Development in Young Adults Study. Circulation 115: 1004-1011. [Crossref]

11. McArthur L, Grady FM, Rosenberg RI, Howard AB (2000) Knowledge of college students regarding three themes related to dietary recommendations. American Journal of Health Studies 16.
12. Blackburn GL (2005) Teaching, learning, doing: best practices in education. Am J Clin Nutr 82: 218S-221S. [Crossref]

13. Zoellner J, Connell CL, Santell R, Fungwe T, Strickland E, et al. (2007) Fit for Life Steps: Results of a community walking intervention in the rural Mississippi Delta. Progress in Community Health Partnerships: Research, Education and Action 1: 49 60. [Crossref]

14. Racette SB, Deusinger SS, Strube MJ, Highstein GR, Deusinger RH (2005) Weigh changes, exercise, and dietary patterns during freshman and sophomore years of college. Journal of American College Health 53: 245-251. [Crossref]

15. Kumanyika S (2006) Nutrition and chronic disease prevention: priorities for US minority groups. Nutr Rev 64: S9-14. [Crossref]

16. Prochaska JO, Johnson S, Lee P (1998) The transtheoretical model of behavior change. In: Shumaker, S.A., Schron, E.B., Ockene, J.K., McGee, W.L., editors. The Handbook of Health Behavior Change. (2nd Edn.), New York, NY: Springer Publishing: 59-84.

17. Lim JW, Gonzalez P, Wang-Letzkus MF, Ashing-Giwa KT (2009) Understanding the cultural health belief model influencing health behaviors and health-related quality of life between Latina and Asian-American breast cancer survivors. Support Care Cancer 17: 1137-1147. [Crossref]

18. Airhihenbuwa C (2010) Culture matters in global health. European Health Psychologist 12: $52-55$.

19. Bureau B (2011) Changing unhealthy eating habits: a reminder-cue based intervention among Moroccan women in Amsterdam.

20. Davis S (2008) The influence of collectivistic and individualistic value orientations on the acceptance of individually-tailored internet communications. The Journal of Education, Community, and Value 8.

21. Dinger MK, Waigandt A (1997) Dietary intake and physical activity behaviors of male and female college students. Am J Health Promot 11: 360-362. [Crossref]

22. Haberman S, Luffey D (1998) Weighing in college students' diet and exercise behaviors. J Am Coll Health 46: 189-191. [Crossref]

23. Anding JD, Suminski RR, Boss L (2001) Dietary intake, body mass index, exercise, and alcohol: are college women following the dietary guidelines for Americans? $\mathrm{J} \mathrm{Am}$ Coll Health 49: 167-171. [Crossref]

24. Desai MN, Miller WC, Staples B, Bravender T (2008) Risk factors associated with overweight and obesity in college students. $J$ Am Coll Health 57: 109-114. [Crossref]

25. Huang TT, Harris KJ, Lee RE, Nazir N, Born W, et al. (2003) Assessing overweight, obesity, diet, and physical activity in college students. $J$ Am Coll Health 52: 83-86. [Crossref]

26. Kimm SY, Glynn NW, Kriska AM, Fitzgerald SL, Aaron DJ, et al. (2000) Longitudinal changes in physical activity in a biracial cohort during adolescence. Med Sci Sports Exerc 32: 1445-1454. [Crossref]

Copyright: (C2015 Gao-Balch YH. This is an open-access article distributed under the terms of the Creative Commons Attribution License, which permits unrestricted use, distribution, and reproduction in any medium, provided the original author and source are credited. 